\title{
Solar atlas revised ${ }^{\star}$
}

\author{
P. Molaro and S. Monai \\ Osservatorio Astronomico di Trieste, via G. B. Tiepolo 11, 34131 Trieste, Italy \\ e-mail:molaro@oats.inaf.it \\ Received 19 December 2011 / Accepted 18 February 2012 \\ ABSTRACT \\ Context. Present knowledge of the solar spectrum is limited because it is very difficult to observe the integrated solar spectrum at high \\ resolution. The reflected solar light from asteroids has been shown to provide a relatively straightforward integrated and unmodified \\ solar spectrum. \\ Aims. We exploit this methodology to improve our knowledge of solar photospheric line positions both in terms of line number and \\ precision with respect to the available solar line atlas. \\ Methods. We used the DAOSPEC program to measure solar line positions automatically in high signal-to-noise spectra of Ceres \\ obtained with HARPS at the $3.6 \mathrm{~m}$ ESO telescope. The line positions were then verified with the solar HARPS spectra of Ganymede \\ and daylight as well as with a new FTS solar spectra. \\ Results. A new atlas with 2334 lines in the range 4000-6859 A is provided. The new atlas is consistent with that based on FTS solar \\ spectra but the new line list improves the precision of individual lines by at least a factor 3-5. \\ Conclusions. The new atlas provides a benchmark for 3D models of the solar photosphere and can be used to trace possible line drifts \\ related to solar activity or to calibrate space and ground spectrographs.
}

Key words. Sun: photosphere - Sun: fundamental parameters - atlases - reference systems - stars: atmospheres

\section{Introduction}

The solar spectrum is a primary reference for stellar astrophysics and for interpreting physical processes in stars, in particular for less evident processes such as differential rotation, convection and magnetic field surface inhomogeneities and their variation during activity cycles. Solar atlases are mostly assembled from observations of center regions of the solar disk, while solar spectra taken over the integrated disk are very rare. The best-quality solar spectra available in the optical and infrared are those obtained at the McMath-Pierce Solar Telescope at Kitt Peak National Observatory with the Fourier Transform Spectrometer (FTS) by Kurucz et al. (1984) and Brault \& Neckel (1987). These atlases comprise a set of observations performed from November 1980 to June 1981. The spectrum covers the spectral region from 296 to $1300 \mathrm{~nm}$ and achieves a signal-to-noise ratio of $\approx 2500$ with a resolving power of $R \approx 400000$. Recently, these observations were fully reprocessed by Kurucz (2005). Despite these exquisite solar spectra, the observed wavelengths of the solar lines are known with a precision at the level of a few hundred $\mathrm{m} \mathrm{s}^{-1}$ at best (Allende Prieto \& Garcia Lopez 1998a,b, hereafter APGL). The FTS solar spectrum is a merger of several observations taken at different positions of the solar disk that have been combined to produce a solar flux of the integrated surface. Single FTS observations required long integrations and were taken at different airmass and different radial velocity drifts because of the Earth's rotation.

A considerable effort was made to evade the problem posed by the extended disk of the Sun. To observe disk-integrated sunlight very few NSO FTS solar spectra were taken with the

* Full Table 3 is only available at the CDS via anonymous ftp to cdsarc.u-strasbg.fr $(130.79 .128 .5)$ or via

http://cdsarc.u-strasbg.fr/viz-bin/qcat?]/A+A/544/A125 un-focused sunlight (Gray \& Livingston 1997; Wallace et al. 2011). At Sacramento Peak Observatory, Beckers et al. (1976) used a cylindrical lens of $5 \mathrm{~mm}$ focal length to achieve a line image of the solar disk in the spectrograph. Other observers following Wallerstein (1962) observed the Moon, Jupiter's moons, or asteroids to obtain the integrated solar spectrum.

Asteroids are an economical way to derive solar integrated flux. They have no intrinsic emission spectrum, they are slow rotators, and their shapes, at least that of the large ones, are almost spherical. Therefore, they reflect almost unaltered sunlight, providing spectra that can be considered good replicas of the solar integrated spectrum (Zwitter et al. 2007; Molaro et al. 2008a,b). Because asteroid kinematics can be computed with high accuracy, they are also good radial velocity standards, and as such they will be used in the coming Gaia mission (Crifo et al. 2007). The radial velocity of an astronomical source is not a trivial quantity. It involves considering i) the mechanism of the light emission; ii) the estimation of the motion of the source and the light propagation through varying gravitational fields and expanding space; iii) the motion of the observer; and iv) the spectrograph used to record the spectrum (Lindegren \& Dravins 2003).

Photospheric lines are built up of many contributions from a variety of temporally variable inhomogeneities across the stellar surface that are connected to solar activity. Moreover, their positions depend on the accuracy of the wavelength calibration of conventional cross-dispersed spectrographs. The laboratory wavelengths of the hollow cathode thorium lamps and iodine absorption cells used for spectrometer calibration are known with a precision that varies from about $15 \mathrm{~m} \mathrm{~s}^{-1}$ to more than $100 \mathrm{~m} \mathrm{~s}^{-1}$, depending on the line strength (Murphy et al. 2007; Lovis \& Pepe 2007). Wilken et al. (2010) found that $\mathrm{CCD}$ manufacturing defects might be responsible for an 
Table 1. Journal of asteroids observations.

\begin{tabular}{|c|c|c|c|c|c|c|c|c|c|c|}
\hline Target & Date & $\begin{array}{c}\text { ESO-JD } \\
(245+)\end{array}$ & $\begin{array}{l}\text { Visual } \\
\text { mag }\end{array}$ & $\begin{array}{l}\text { Exp. } \\
\text { (s) }\end{array}$ & $\begin{array}{c}\mathrm{RV} \\
\text { (ast-Obs.) }\end{array}$ & $\begin{array}{c}\mathrm{RV} \\
\text { (ast- } \odot \text { ) }\end{array}$ & $\begin{array}{c}\Delta \mathrm{RV} \\
\mathrm{km} \mathrm{s}^{-1}\end{array}$ & $\begin{array}{l}\text { BERV } \\
\mathrm{km} \mathrm{s}^{-1}\end{array}$ & $\begin{array}{l}\mathrm{RV} \exp \\
\mathrm{km} \mathrm{s}^{-1}\end{array}$ & N. Spots \\
\hline Ceres & $2006-07-15$ & 3932.8421400 & 8.0 & 1800 & -11.288 & 0.456 & -10.832 & 14.754 & 3.922 & 9 \\
\hline Ganymede & 2007-04-13 & 4203.4030533 & 5.1 & 300 & -21.818 & 2.800 & -19.018 & 24.956 & 5.938 & 0 \\
\hline Vesta & $2009-12-23$ & 5188.3410970 & 7.3 & 300 & -21.946 & -1.512 & -23.457 & 27.292 & 3.835 & 0 \\
\hline Vesta & $2009-12-24$ & 5189.3733994 & 7.3 & 300 & -21.691 & -1.515 & -23.206 & 26.980 & 3.774 & 0 \\
\hline Vesta & $2009-12-25$ & 5190.3628972 & 7.3 & 300 & -21.541 & -1.519 & -23.060 & 26.777 & 3.716 & 0 \\
\hline
\end{tabular}

Notes. Radial velocities are in $\mathrm{km} \mathrm{s}^{-1}$, BERV is the geocentrical correction applied by the HARPS pipeline, which needs to be added to $\triangle$ RV to obtain the expected radial velocity value.

adaptation of the dispersion curves in the wavelength calibration analysis, which produces distortion at the level of about $\pm 40 \mathrm{~m} \mathrm{~s}^{-1}$ at a specific wavelength. Every solar atlas may contain such inaccuracies, which are unavoidable at the moment. Only the advent of more precise calibration tools such as the laser comb will allow substantial progress.

Molaro \& Centurion (2011) used solar light reflected by the asteroid Ceres to improve the precision of the line position of the Allende Prieto \& Garcia Lopez (1998a,b) line list, but for a rather limited number of lines. In the present paper we analyze HARPS observations of Ceres, Ganymede, and Vesta as well as daylight solar spectrum and the new FTS solar spectra provided by Kurucz (2005) and Wallace et al. (2011) to provide a highprecision solar atlas with an increased number of lines.

\section{The integrated disk solar data}

\subsection{Kurucz 2005 and Wallace et al. 2011}

To observe disk-integrated sunlight, a few NSO FTS solar spectra were taken from an out-of-focus image of the Sun ${ }^{1}$. The beam was directed to the $0.5 \mathrm{~mm}$-wide entrance slit of the spectrograph by a flat mirror which substituted the usual concave focusing mirror of the telescope. This aperture acted as a pinhole camera, imaging the Sun on the spectrograph (Gray \& Livingston 1997; Wallace et al. 2011). There are notable differences in line depth and width between flux and disk-center spectra, the latter being narrower and with a greater line depth (cf. Fig. 1 in Wallace et al. 2011). Integrated light FTS spectra were observed on only two occasions. The first was in 1980-1981 and was used in the Kurucz et al. (1984) atlas and in 1989 to study changes in the solar irradiance over the solar cycle (Mitchell \& Livingston 1991).

A revised spectrum of the FTS Kitt Peak Solar Flux Atlas for 300 to $1000 \mathrm{~nm}$ was produced by Kurucz (2005). About fifty solar FTS scans were taken by J. Brault and L. Testerman at Kitt Peak. Eight scans are required to cover the whole spectral range and several others for every portion of the spectral region to reproduce the integrated solar spectrum. The flux spectrum from 0.3 to $1.3 \mu \mathrm{m}$ was published as the Solar Flux Atlas by Kurucz et al. (1984). However, each FTS scan has been taken in different epochs and has its own air mass, and radial velocity, which affect the merged spectrum. The frequency scale for each FTS scan was determined by observing the $\mathrm{O}_{2} 688.38335 \mathrm{~nm}$ line through a gas absorption cell. However, the $\mathrm{O}_{2}$ line is present only in scan 1 . The other scans were calibrated by aligning the solar lines in overlapping regions or by using telluric

\footnotetext{
1 A history of the McMath-Pierce solar telescope and of the FTS by Claude Plymate can be found at http://nsokp.nso.edu/mp/ cphistory.html
}

lines when available. The wavelengths are aligned with the observations taken on 24 March 1981. Kurucz et al. (1984) estimated the error on the zero scale to be about $100 \mathrm{~m} \mathrm{~s}^{-1}$ and this likely remains also in the new reduction since there are no new elements that may have improved it.

The integrated solar spectra taken in 1989 form the solar flux spectra recently provided by Wallace et al. (2011). The optical region from 2958 to $9257 \AA$ is covered by six spectral regions, each limited by optical band pass filters. The $\mathrm{S} / \mathrm{N}$ in the continuum is several hundreds and the resolving power reaches $\approx 700000$. The McMath-Pierce FTS operates in vacuum but wavelengths have been provided in air for STP conditions. Wallace et al. (2011) have corrected their spectra by measuring the FeI line positions and by shifting them to those of Nave et al. (1994). In this way they have removed Earth motions and gravitational redshifts. However, because the FeI lines suffer for different convective shifts and the correction depends on the specific set of lines considered, the doppler correction is not an objective quantity. In their analysis of the FTS spectrum Wallace et al. (2011) noted that "numerous $S$ structures in the transmission spectrum appeared due to the doppler shift between the solar lines when the telluric lines were matched up" and that different stretchings were needed for $\mathrm{H}_{2} \mathrm{O}$ and $\mathrm{O}_{2}$ telluric regions. Moreover, the A-D converter is somewhat non-linear producing an error in the zero level and in line depths. This may also affect the wavelengths of blended lines. Wallace used saturated atmospheric lines to adjust the zero with an extrapolation in the regions such as the blue where saturated lines are absent.

Both sets of observations (1980-1981 and 1989) were taken near a peak of solar activity. The sunspot numbers were 177 and 130 for the 1980 and 1981 observations, respectively, and of 129 and 159 in the observations of 1 and 13 of October 1989 respectively. This means that the FTS spectra refer to a period of high solar activity.

\subsection{Asteroids and Ganymede}

We analyzed several observations of asteroids made with the HARPS spectrograph, which are available from the public HARPS archive. These observations are reported in Table 1 together with some basic information. HARPS spectra are taken with a resolving power of $R=\lambda / \delta \lambda=115000$ and the 90A is sampled with 4.1 pixels $15 \mu$ wide and simultaneously covers the range from 380 to $690 \mathrm{~nm}$, leaving only a small gap at $530-539 \mathrm{~nm}$. The fiber feeding the spectrograph is equipped with an image scrambler to provide a uniform spectrograph pupil illumination. This is an important difference compared to slit spectrographs where non-uniform slit illumination induces radial velocity differences between different exposures. HARPS 
was able to deliver a sequence of observations over a 500-day baseline with a dispersion of $64 \mathrm{~cm} \mathrm{~s}^{-1}$ along the exoplanet orbit.

We first reconsidered the HARPS spectrum of Ceres that was analyzed by Molaro \& Centurion (2011). Ceres is the largest asteroid with a spherical shape and a radius of $476.2 \mathrm{~km}$. The rotation is $\approx 91 \mathrm{~m} \mathrm{~s}^{-1}$ and is therefore negligible compared to that of the Sun.

Vesta is one of the brightest asteroids and therefore can provide a high signal-to-noise ratio from relatively short exposures. To our knowledge, Vesta was the first asteroid to be observed to obtain a solar spectrum that was then used in the analysis of stellar spectra (Wallerstein 1962). Here we have considered a series of three observations of Vesta taken during successive nights to check if we are able to track the daily change in radial velocity.

Ganymede, one of the Jupiter's moons, is particularly bright and provides quite high $\mathrm{S} / \mathrm{N}$ spectra in a relatively short time. It has a radius of $2634 \mathrm{~m}$ and a synchronous rotation period of $7.15 \mathrm{~d}$. At the epoch of observations it had a magnitude of $\approx 5 \mathrm{mag}$ and angular dimensions of $1.5 \mathrm{arcsec}$. The rate of change in the radial velocity was $9 \mathrm{~m} \mathrm{~s}^{-1}$ every minute, but the exposures took only five minutes.

In contrast with the FTS spectra of the Sun, the asteroid observations analyzed here were made during a minimum of activity of the solar cycle. The number of spots at that epoch is reported in the last column of Table 1 and corresponds to zero or is a very low number.

\subsection{Sky-light}

A daylight solar spectrum obtained from a combination of more than 30 spectra was provided by Dall et al. (2006). The nominal $\mathrm{S} / \mathrm{N}$ is greater than 1000 , but effective measurements in the spectrum yielded only about half of that. Molaro et al. (2008a,b) showed that sky spectra are sensitive to unpredictable motions likely caused by currents in the upper terrestrial atmosphere and therefore do not provide the perfect rest velocity. Close inspection of UVES twilight and asteroid solar spectra show that there are small differences at the level of $1-2 \%$ between them. These differences were found to depend also on the angular separation from the Sun (Hunten 1970; Gray et al. 2000). The FWHM of the lines of the daylight spectrum are higher compared to those of the asteroids suggesting the presence of turbulence in the atmospheric layers that reflect sunlight. At twilight, a transverse motion in the atmosphere has a considerable radial velocity component in the direction of the Sun and produces a drift in the reflected spectrum.

Although the twilight spectrum is not a good zero reference point, we used it and took advantage of the high signal-to-noise ratio.

\section{Methodology}

The first solar atlas obtained by means of asteroid observations was provided by Molaro \& Centurion (2011). This first pilot work consisted of by a set of about 800 solar lines measured individually. Here we used the DAOSPEC program on the same solar spectra to increase the number of lines. DAOSPEC, developed by Stetson, is a program that automatically finds absorption lines in stellar spectra, fits the continuum and identifies lines with the help of a laboratory line list (Stetson \& Pancino 2008). Of interest here is that DAOSPEC provides the position and dispersion of the lines around the mean radial velocity together with a mean radial velocity estimate from the lines identified in the

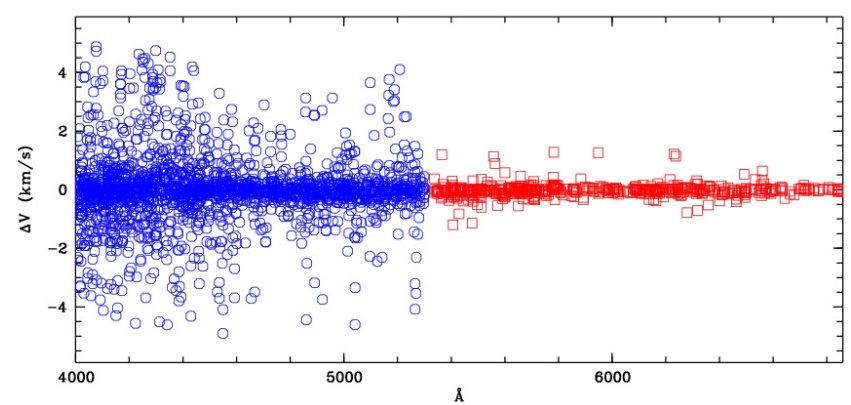

Fig. 1. Velocity difference between the APGL lines and those obtained by DAOSPEC from the Kurucz (2005) FTS solar spectrum.

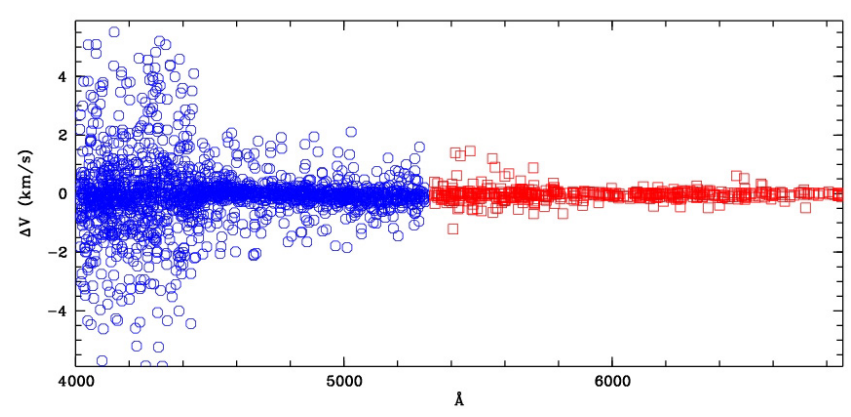

Fig. 2. Velocity difference between the APGL lines and those obtained by DAOSPEC from the Wallace et al. (2011) FTS solar spectrum.

spectrum. We used DAOSPEC on different kinds of integrated solar spectra at our disposal after normalization. We established a minimum equivalent width threshold of $25 \AA$ to avoid problems with relatively weak lines and performed the analysis on the two different HARPS arms separately. This naturally identifies a blue range, which we set to begin at $400 \mathrm{~nm}$ to exclude the region of the strong CaII lines up to $530 \mathrm{~nm}$ and red spectrum between $533.5 \mathrm{~nm}$ up to $680.0 \mathrm{~nm}$. These two ranges have quite significant differences in terms of the number and density of solar photospheric lines. As our initial line list we used the one by APGL, which provides many lines with a fairly good spectral coverage.

\section{Results}

We first analyzed the revised FTS spectrum provided by Kurucz (2005) with DAOSPEC. The mean radial velocity of the spectrum is $-0.515 \mathrm{~km} \mathrm{~s}^{-1}$ in the red and $-0.552 \mathrm{~km} \mathrm{~s}^{-1}$ in the blue. According to Kurucz (2005), the solar spectrum has been shifted to correct for the gravitational redshift. The correction made by Kurucz is not reported in his paper, but the measured radial velocity is consistent with a gravitational redshift of $636.5 \mathrm{~m} \mathrm{~s}^{-1}$ and an uncertainty in the original absolute scale of $\approx 100 \mathrm{~m} \mathrm{~s}^{-1}$. However, the dispersion of the line position is considerable, with standard deviations of 0.260 and $0.986 \mathrm{~km} \mathrm{~s}^{-1}$ in the red and in the blue, respectively. The differences between the line positions found by DAOSPEC in this solar spectrum and the APGL measurements are shown in Fig. 1. The differences are increasing toward shorter wavelengths, where they reach values of $\mathrm{km} \mathrm{s}^{-1}$. A similar indication is provided by the analysis of the Wallace et al. (2011) solar spectrum (Fig. 2).

The analysis of the solar spectrum provided by Kurucz et al. (1984), which is the spectrum used by APGL to produce their line list, had shown a slightly reduced, but still considerable scatter. This suggests that differences in the line position originate in 


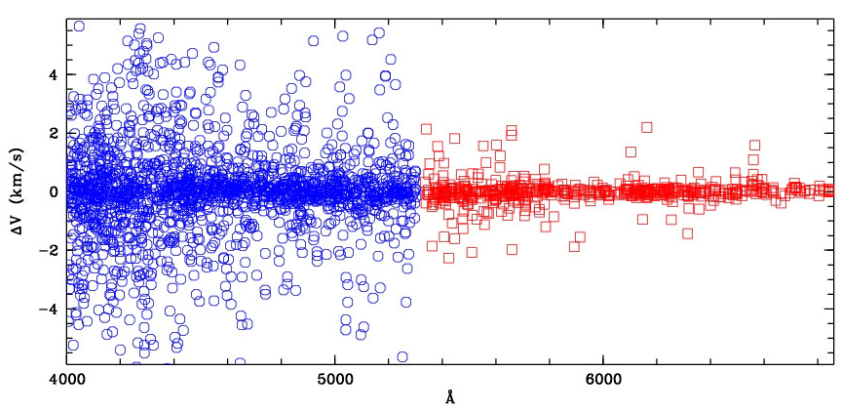

Fig. 3. Velocity difference between the APGL lines and those obtained by DAOSPEC from Ceres solar spectrum.

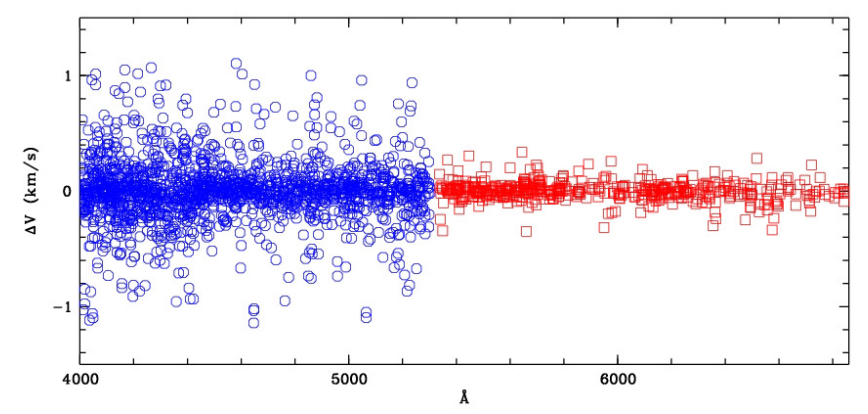

Fig. 4. Velocity difference between the lines of Table 3 and those measured with DAOSPEC in Ganymede's solar spectrum. Note the different $y$ scale compared to previous figures.

the different processes of measuring the line position between DAOSPEC and APGL. In particular, APGL did not identify blends of lines, and the line position given is in fact the blend centroid. DAOSPEC instead is guided by the FWHM of the lines and rejects the lines that exceed the expected line width. This also explains why the deviations are increasing toward the blue, where the lines are more crowded and blends more common.

We then analyzed the HARPS spectrum of Ceres with DAOSPEC. The expected radial velocity was computed with the JPL ephemerides for the mid-exposure exact time following the method used in Molaro et al. (2008a,b) and is nominally at the level of $\mathrm{cm} \mathrm{s}^{-1}$. For this epoch the expected radial velocity of Ceres is $3.922 \mathrm{~km} \mathrm{~s}^{-1}$, while the analysis with DAOSPEC provides a radial velocity of $3.911 \mathrm{~km} \mathrm{~s}^{-1}$ in the red portion which agrees well with the computed velocity. The order of magnitude of the error of the radial velocity is $\approx 10 \mathrm{~m} \mathrm{~s}^{-1}$ as estimated from the typical error of the measure of a line divided by the square root of the number of lines. The blue region provides a radial velocity of 3.961 which agrees slightly less well than expected, but despite more lines in the blue, the error of the radial velocity is about three times larger because of the much higher dispersion.

The scatter of the lines around the mean velocity provided by DAOSPEC is also high for Ceres. The observed dispersion is $0.422 \mathrm{~km} \mathrm{~s}^{-1}$ and $1.483 \mathrm{~km} \mathrm{~s}^{-1}$ for the red and blue lines, respectively, as shown in Fig. 3, which agrees reasonably well with the dispersion observed with the FTS solar spectrum. This confirms that the origin lies not in the FTS solar spectrum but in the line list provided by APGL. Therefore, we used the line positions provided by DAOSPEC in the spectrum of Ceres to construct a new atlas of solar line positions.

The new list is the second column of the output of DAOSPEC and is given in Table 3. The final line list comprises 2088 and 822 lines in the ranges 4000-5304 and 5335-6859 $\AA$, respectively. These positions have been slightly corrected to

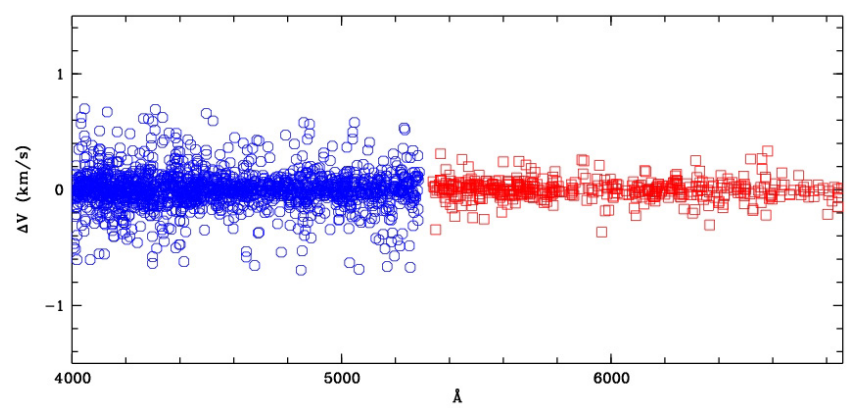

Fig. 5. Velocity difference between the lines of Table 3 and those measured with DAOSPEC in the 25 Dic Vesta solar spectrum.

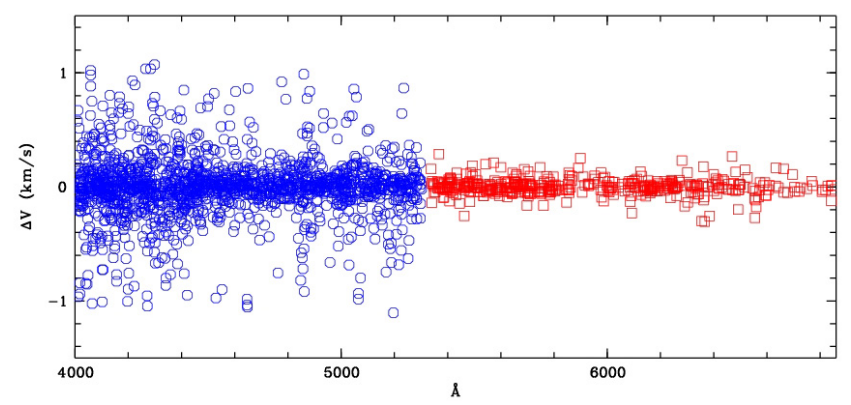

Fig. 6. Velocity difference between the lines of Table 3 and those measured with DAOSPEC in the daylight solar spectrum.

match the observed radial velocity with the expected one, which we consider more reliable. Namely we shifted the lines in the blue region by $-39.9 \mathrm{~m} \mathrm{~s}^{-1}$ and the lines in the red portion by $+11.1 \mathrm{~m} \mathrm{~s}^{-1}$. The positions correspond to air wavelengths with the identification taken from APGL. The table provides only the first entries but the full list is available electronically.

We have checked this new line list against the other available integrated solar spectra. The new list on the Kurucz (2005) solar spectrum provides global radial velocities of -0.556 and $-0.624 \mathrm{~km} \mathrm{~s}^{-1}$, which are slightly closer to the nominal gravitational correction applied to the spectrum. The dispersion decreases in the red portion but remains high, which implies that the stretchings of the FTS spectral reduction are still present. When we used the new line list to analyze the spectra of the asteroids, we obtained a considerable decrease of the dispersion around the mean radial velocity. For instance, in the blue portion of Jupiter's moon Ganymede the dispersions are only $0.095 \mathrm{~km} \mathrm{~s}^{-1}$ and $0.077 \mathrm{~km} \mathrm{~s}^{-1}$ in the blue and red portions, respectively, as shown in Fig. 4. With the skylight spectrum the dispersions are $0.122 \mathrm{~km} \mathrm{~s}^{-1}$ and $0.065 \mathrm{~km} \mathrm{~s}^{-1}$ in the blue and in the red, respectively (Fig. 5). In the three Vesta spectra the dispersion is $\approx 0.080 \mathrm{~km} \mathrm{~s}^{-1}$ and $\approx 0.140 \mathrm{~km} \mathrm{~s}^{-1}$ in the red and in the blue, respectively, showing a high reproducibility of the results among the three observations. Figure 6 shows the case for the Vesta observation of 25 December 2009. These values are close to the mean error of the individual positions as estimated by Molaro \& Centurion (2011) and they can be accounted for entirely by photon noise. The fact that the analysis always returns excellent results for a variety of integrated solar spectra makes us confident that the new line list we have provided identifies the line positions correctly.

Where they are the same as in Molaro \& Centurion (2011), the new line positions agree well. In Fig. 7 the lines measured with DAOSPEC in Ceres are compared with the position measurements provided in Molaro \& Centurion (2011). The 
Table 2. DAOSPEC analysis of different solar spectra.

\begin{tabular}{lccccccccc}
\hline \hline Target & $\begin{array}{c}\mathrm{RV}_{\text {red }} \\
\mathrm{km} \mathrm{s}^{-1}\end{array}$ & $\begin{array}{c}\sigma \\
\mathrm{km} \mathrm{s}^{-1}\end{array}$ & $\mathrm{~N}$. lines & $\begin{array}{c}\mathrm{RV}_{\text {blue }} \\
\mathrm{km} \mathrm{s}^{-1}\end{array}$ & $\begin{array}{c}\sigma \\
\mathrm{km} \mathrm{s}^{-1}\end{array}$ & $\mathrm{~N}$. lines & Input lines & $\begin{array}{c}\Delta \mathrm{RV}(o-e)_{\text {red }} \\
\mathrm{m} \mathrm{s}^{-1}\end{array}$ & $\begin{array}{c}\Delta \mathrm{RV}(o-e)_{\text {blue }} \\
\mathrm{m} \mathrm{s}^{-1}\end{array}$ \\
\hline FTS-2005 & -0.515 & 0.260 & 1208 & -0.552 & 0.986 & 2682 & APGL & -122 & -84 \\
FTS-1984 & 0.006 & 0.222 & 1189 & 0.005 & 0.572 & 2647 & APGL & - & - \\
FTS-2011* & -0.221 & 0.292 & 450 & -0.157 & 1.357 & 1043 & APGL & -84 & 143 \\
FTS-2011** & -0.322 & 0.173 & 672 & -0.173 & 0.436 & 1523 & APGL & 34 & -36 \\
Ceres & 3.911 & 0.422 & 1217 & 3.962 & 1.483 & 2386 & APGL & -11 & 40 \\
FTS-2005 & -0.537 & 0.115 & 464 & -0.645 & 1.017 & 1798 & this paper & -99 & 8 \\
Ganymede & 5.9384 & 0.070 & 501 & 5.9386 & 0.232 & 1713 & this paper & 0.4 & 0.6 \\
Vesta (23) & 3.843 & 0.079 & 488 & 3.836 & 0.140 & 1714 & this paper & 8 & 1 \\
Vesta (24) & 3.783 & 0.084 & 495 & 3.779 & 0.141 & 1723 & this paper & 9 & -2 \\
Vesta (25) & 3.714 & 0.074 & 488 & 3.709 & 0.140 & 1701 & this paper & -2 & -7 \\
SKY & -0.026 & 0.061 & 480 & -0.086 & 0.220 & 1712 & this paper & -26 & -86 \\
\hline
\end{tabular}

Notes. ${ }^{(*)}$ Refers to the Wallace et al. (2011) spectrum in the ranges 5335-5747 and 4015-4443 $\AA$. ${ }^{(* *)}$ The same, but for the ranges $5747-6859$ and 4443-5304 ̊.

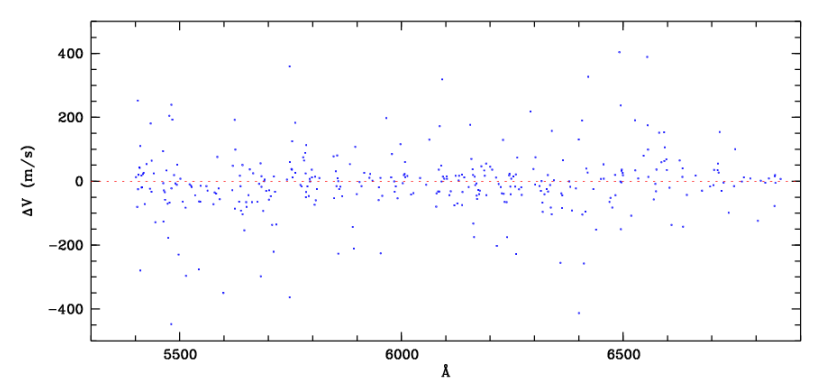

Fig. 7. Velocity differences between the lines of Table 3 and the line positions provided in Molaro \& Centurion (2011).

substantial agreement between the two sets of measures is evident although they were obtained with different procedures. This is confirmed from the distribution histogram of these data shown in Fig. 8. As shown in Molaro \& Centurion (2011), the typical errors in the single lines are $\approx 45 \mathrm{~m} \mathrm{~s}^{-1}$ and $90 \mathrm{~m} \mathrm{~s}^{-1}$ in the blue where the signal-to-noise ratio is lower and the Ceres spectrum provides at least a factor three improvement with respect to the APGL measurements.

Because the Ceres spectrum was taken during a solar activity minimum, as indicated by the few spots over the stellar surface, implies that our line positions refer to those of the quiet sun, which is different from those of the APGL list, which were derived from a solar spectrum taken during a magnetic activity peak.

The comparison between the expected radial velocity and that actually measured in the spectra is given in the last two columns of Table 2 for the red and blue portions considered separately. For the FTS solar spectra the difference is between the gravitational redshift of $636.5 \mathrm{~m} \mathrm{~s}^{-1}$ and the observed one. What we are measuring is the correction Wallace et al. (2011) applied to the spectra. In this case we considered portions that belong to the different FTS scans that compose the solar spectrum of these authors. This is because Wallace et al. (2011) applied different radial velocity corrections to the different spectral scans. However, the wavelength ranges do not match precisely and radial velocities are sensitive to the specific lines used. Accordingly our radial velocities cannot be the same as the corrections of Wallace et al. (2011) although they are fairly close. In the particular case of Vesta, three exposures were made in three successive nights and the daily drift is about $60 \mathrm{~m} \mathrm{~s}^{-1}$.

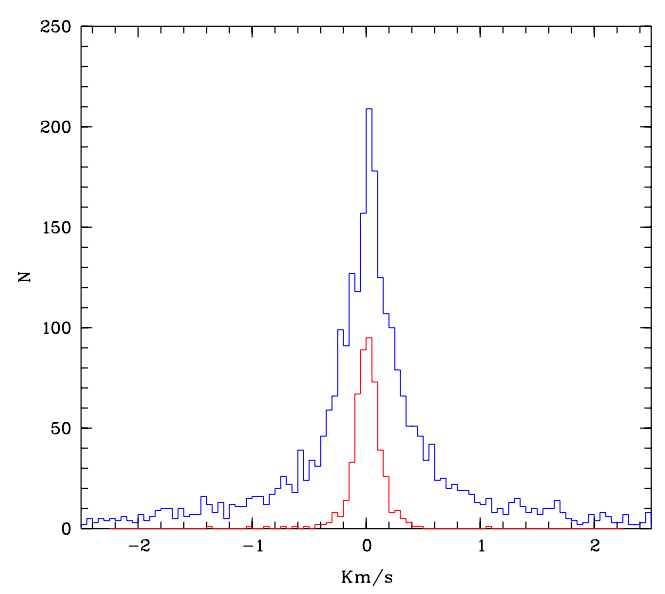

Fig. 8. Histogram of velocity distribution of Fig. 7, the external (blue) line is relative to the present analysis and the internal (red) line to that of Molaro \& Centurion (2011).

We were able to follow the radial velocity drift and match the absolute radial velocity within few $\mathrm{m} \mathrm{s}^{-1}$. We emphasize that this is quite different from what is currently done in the search for exoplanets where the spectra are used to detect differential drifts at sub- $\mathrm{m} \mathrm{s}^{-1}$ level, regardless of the absolute value of radial velocity.

\subsection{Analysis of the neutral iron lines}

Very many of the measured lines, namely 248 falling in the red, are identified with neutral iron. This sample can be compared with the laboratory measurements for FeI provided by Nave et al. (1994), which has an accuracy of $\geq 20 \mathrm{~m} \mathrm{~s}^{-1}$ in the optical. The results are shown in Fig. 9 not corrected for the gravitational redshift. The comparison shows that the lines are generally highly shifted with shifts exceeding several hundred $\mathrm{m} \mathrm{s}^{-1}$. However, the actual shift is the result of the gravitational redshift and convective blueshift, where the latter is generally the lower. As a rule of thumb, weak lines are formed deep in the stellar photosphere, while strong lines, particularly their cores, are formed in higher layers. Therefore, weak lines show higher blue shifts than strong lines which can be observed in Fig. 9. The lines formed at the top of the photosphere are hardly sensitive to convection and 
Table 3. Solar line list positions.

\begin{tabular}{lccc}
\hline \hline Ceres & $\begin{array}{c}\text { Allende } \\
\AA\end{array}$ & & $\begin{array}{c}\text { DV } \\
\mathrm{km} \mathrm{s}^{-1}\end{array}$ \\
\hline 4006.6964 & 4006.7556 & FE1 & -4.427 \\
4007.2763 & 4007.2796 & FE1 & -0.242 \\
4008.0882 & 4008.0489 & TI1 & 2.940 \\
4008.8991 & 4008.9325 & TI1 & -2.492 \\
4009.6842 & 4009.7225 & FE1 & -2.861 \\
4010.1798 & 4010.1742 & FE1 & 0.424 \\
4010.8640 & 4010.7761 & FE1 & 6.570 \\
4011.4175 & 4011.4111 & FE1 & 0.481 \\
4011.7179 & 4011.7146 & FE1 & 0.250 \\
4012.2036 & 4012.2479 & ND2 & -3.306 \\
4012.4028 & 4012.3903 & TI2 & 0.934 \\
4012.6443 & 4012.6075 & NI1 & 2.752 \\
4013.6274 & 4013.6390 & FE1 & -0.861 \\
4013.8333 & 4013.8193 & FE1 & 1.046 \\
4014.2681 & 4014.2681 & FE1 & 0.007 \\
4014.5302 & 4014.5355 & FE1 & -0.393 \\
4014.9274 & 4014.9327 & CE2 & -0.391 \\
4015.3985 & 4015.3757 & TI1 & 1.706 \\
4016.4228 & 4016.4228 & FE1 & 0.003 \\
4016.8085 & 4016.7987 & V2 & 0.738 \\
4017.1307 & 4017.1520 & FE1 & -1.588 \\
4017.5020 & 4017.4670 & NI1 & 2.612 \\
4017.7700 & 4017.7711 & TI1 & -0.077 \\
4018.1123 & 4018.1051 & MN1 & 0.540 \\
4018.2829 & 4018.2707 & FE1 & 0.917 \\
4019.0544 & 4019.0498 & FE1 & 0.348 \\
4020.0552 & 4020.0582 & FE1 & -0.221 \\
\hline
\end{tabular}

Notes. The full table is available at the CDS.

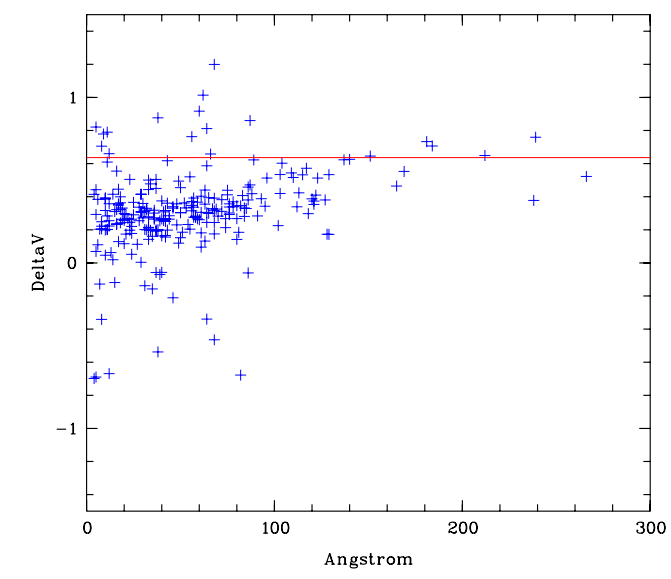

Fig. 9. Convective shifts not corrected for gravitational redshift.

therefore show shifts close to the gravitational redshift, while the other lines reveal convective motions with variable blue shifts.

A known correlation exists between the equivalent width and the line shift. Lines with EWs greater than 150-200 m are quite insensitive to convective shifts and reach a plateau that can be used to estimate the absolute zero of the solar spectrum. Out of our sample only nine lines have an EW greater than $150 \mathrm{~mA}$ and show a mean value of $\approx 601 \pm 43 \mathrm{~m} \mathrm{~s}^{-1}$, which agrees well with the theoretical gravitational shift of $636.5 \mathrm{~m} \mathrm{~s}^{-1}$.

\section{Summary and future prospects}

From the analysis of a high signal-to-noise integrated solar spectrum obtained from asteroids we have provided a new atlas of solar line positions with 2334 entries in the range 400-690 nm. Internal precision of individual line position is $\approx 50 \mathrm{~m} \mathrm{~s}^{-1}$. These improve by a factor 3 but often by one order of magnitude or even more the existing solar atlas of APGL. However, the accuracy ultimately relies on HARPS performances.

The whole atlas can be used to calibrate existing spectrographs at any wavelength in the covered range down to the claimed precision and provides absolute reference at the level of few $1 \mathrm{~m} \mathrm{~s}^{-1}$.

The technique of exploiting the sunlight reflected by asteroids moving by known radial velocity could be be usefully applied to monitor the behavior of convective solar lines along the cycle of magnetic activity through sequences of observations along the solar cycle. Jimenez et al. (1986) measured changes in the radial velocity of the integrated solar spectrum in the period from 1976 and 1985 with a resonant scattering spectrometer and found variations of $16 \mathrm{~m} \mathrm{~s}^{-1}$ in corresponding to the peak cycle. Deming \& Plymate (1994) measured these displacements as a peak-to-peak modulation of $30 \mathrm{~m} \mathrm{~s}^{-1}$ over the 11-year solar activity period with the positions redshifted corresponding to the activity maximum. However, McMillan et al. (1993) did not reveal any drift within $4 \mathrm{~m} \mathrm{~s}^{-1}$ in the solar line position from a long data series spanning from 1987 to 1992. Lagrange et al. (2010, 2011) have computed the radial velocity variations caused by the spots observed on the Sun's surface in cycle 23 (between 1993 and 2003) and included them in a synthetic model of the solar spectrum. They found typical amplitudes of up to a few $\mathrm{m} \mathrm{s}^{-1}$ depending on the activity level and on the temperature of the spot, but they were lower than those observed by Jimenez et al. (1986) and Deming \& Plymate (1994). The periodograms contain peaks at periods that are sometimes very different from the solar rotation period and therefore require an accurate controlling to be revealed. Meunier et al. (2010) considered the presence of plages. Contrary to spots, plages suppress convection in the active regions, leading always to a redshift of the whole solar spectrum. Meunier et al. (2010) found a long-term amplitude of $8 \mathrm{~m} \mathrm{~s}^{-1}$ while the short term radial velocity amplitude is on the order of $\mathrm{m} \mathrm{s}^{-1}$ or down $0.4 \mathrm{~m} \mathrm{~s}^{-1}$ during the low-activity period. Gray (2009) pointed out that the shift is line-dependent and that strong and weak lines should give different shifts, which would be interesting to measure.

These activity-related movements are expected at the level of few $\mathrm{m} \mathrm{s}^{-1}$ and call for higher precision. This atlas offers a significant advancement but can be even more improved both in terms of spectral range and of line position precision. New asteroid observations with HARPS, in particular taken with the laser comb, are therefore solicited.

Acknowledgements. We are grateful to Peter Stetston who made available his code and modified it to increase the number of significant figures in the radial velocity output to match our needs. We thank the HARPS group for making available their archive available and to Simonetta Fabrizio and Gabriella Schiulaz for improving the English.

\section{References}

Allende Prieto, C., \& Garcia Lopez, R. J. 1998a, A\&AS, 129, 41 Allende Prieto, C., \& Garcia Lopez, R. J. 1998b, A\&AS, 131, 431

Beckers, J. M., Bridges, C. A., \& Gilliam, L. B. 1976, A High Resolution Spectral Atlas of the Solar Irradiance From 380 to 700 nm, AFGL-TR-760126 (Hanscom, MA: Air Force Geophysics Laboratory) 
P. Molaro and S. Monai: Solar atlas revised

Brault, J., \& Neckel, H. 1987, Spectral Atlas of Solar Absolute Disk-Averaged and Disk-Center Intensity from 3290 to 12510 A, unpublished (tape-copy from KIS IDL library)

Crifo, F., Jasniewicz, G., Soubiran, C., et al. 2007, SF2A-2007: Proc. Annual meeting of the French Society of Astronomy and Astrophysics held in Grenoble, France, July 2-6, eds. J. Bouvier, A. Chalabaev, \& C. Charbonnel, 459

Dall, T. H., Santos, N. C., Arentoft, T., Bedding, T. R., \& Kjeldsen, H. 2006, A\&A, 454, 341

Deming, D., \& Plymate, C. 1994, ApJ, 426, 382

Gray, D. F. 2009, ApJ, 697, 1032

Gray, D. F., \& Livingston, W. C. 1997, ApJ, 474, 802

Gray, D. F., Tycner, C., \& Brown, K. 2000, PASP, 112, 328

Hunten, D. M. 1970, ApJ, 159, 1107

Kurucz, R. 2005, Mem. S.A.It. Suppl., 8, 189, http: //KURUCZ. HARVARD . EDU/ PAPERS/TRANSMISSION

Kurucz, R. L., Furenlid, I. J., \& Testerman, L. 1984, NOAO Atlas No. 1, The Solar Flux Atlas from 296 to 1300 nm, Sunspot (NM: National Solar Observatory)

Jimenez, A., Palle, P. L., Regulo, C., et al. 1986, AdSpR, 6, 89

Lagrange, A.-M., Desort, M., \& Meunier, N. 2010, A\&A, 512, A38
Lagrange, A.-M., Desort, M., \& Meunier, N. 2011, A\&A, 528, L9 Lindegren, L., \& Dravins, D. 2003, A\&A, 401, 1185

Lovis, C., \& Pepe, F. 2007, A\&A, 468, 1115

McMillan, R. S., Moore, T. L., Perry, M. L., \& Smith, P. H. 1993, ApJ, 403, 801

Meunier, N., Desort, M., \& Lagrange, A.-M. 2010, A\&A, 512, A39

Mitchell, W. E., Jr., \& Livingston, W. C. 1991, ApJ, 372, 336

Molaro, P., \& Centurión, M. 2011, A\&A, 525, A74

Molaro, P., Levshakov, S. A., Monai, S., et al. 2008a, A\&A, 481, 559

Molaro, P., Reimers, D., Agafonova, I. I., \& Levshakov, S. A. 2008b, EpJ, 163, 173

Murphy, M. T., Tzanavaris, P., Webb, J. K., \& Lovis, C. 2007, MNRAS, 378, 221

Nave, G., Johansson, S., Learner, R. C. M., Thorne, A. P., \& Brault, J. W. 1994, ApJS, 94, 221

Stetson, P. B., \& Pancino, E. 2008, PASP, 120, 1332

Wallace, L., Hinkle, K., Livingston, W. C., \& Davis, S. P. 2011, N. S. O. Technical Report N. 11-001 (National Solar Observatory), PO Box 26732, Tucson AZ 85726

Wallerstein, G. 1962, ApJS, 6, 407

Wilken, T., Lovis, C., Manescau, A., et al. 2010, MNRAS, 405, 16

Zwitter, T., Mignard, F., \& Crifo, F. 2007, A\&A, 462, 795 